The inclusion of converted cases in the thoracotomy cohort would bias against the thoracotomy approach if converted cases represented those at a disproportionately high risk for postoperative complications. We therefore isolated the multiple approach patients (which include the converted cases) to evaluate the complication rate in this subset of patients. The complication rate in this multiple approach cohort was $38.5 \%$, which was similar to that in patients undergoing thoracotomy alone $(35.9 \% ; P=.071)$ but higher than that in patients undergoing VATS alone $(28.9 \% ; P<.001)$. Converted cases thus do not appear to pose a disproportionately high risk for postoperative complications, and eliminating them from the thoracotomy cohort would not eliminate the study findings that VATS was associated with fewer complications than thoracotomy.

The exclusion of converted cases from the VATS cohort could bias in favor of VATS if the conversions were in response to VATS-specific intraoperative errors (mistakes that could have been avoided by a planned thoracotomy). Intraoperative errors can be directly and indirectly associated with postoperative complications (longer operating room times, intraoperative transfusions associated with postoperative complications). To adjust for this possibility, we combined the multiple approach patients with the VATS cohort. This would not be an intention-to-treat analysis (because not all multiple approach patients had conversions; some underwent planned diagnostic VATS followed by planned thoracotomy). The complication rate of the VATS combined group (multiple approach plus VATS only) was $31.12 \%$, which remains significantly lower than that of the thoracotomyonly group $(35.86 \% ; P<.0001)$. Inclusion of converted cases with the VATS cohort thus does not eliminate the study findings that VATS was associated with fewer complications than thoracotomy.

We therefore believe that the more detailed evaluation of the impact of the multiple approach patients, although an imperfect reflection of conversion, supports our assertion that the safety benefits of VATS are not an artifact of unadjusted conversion bias.

Unexpected pneumonectomy is not specified in the Society of Thoracic Surgeons database, and a disproportionate incidence would be of obvious concern. As with any significant variation in surgical approach, it is reasonable to assume the existence of a set of complications of varying severity (including catastrophic) that are directly attributable to the VATS approach (and potentially avoidable by thoracotomy). Much like common bile duct injuries with the adoption of the laparoscopic cholecystectomy, data resources must expand to capture these approach-specific complications. A more detailed understanding of the prevalence of these complications could motivate an evolution to account for these pitfalls (eg, converting challenging cases before an otherwise unwarranted pneumonectomy becomes inevitable).

Propensity matching is only able to adjust for variables that are included in the match. That being said, many of the variables that characterize the VATS candidacy are captured by the Society of Thoracic Surgeons and were included in the matching (habitus, previous thoracic surgery, comorbidities, laterality, lobar location, etc). There are, however, a number of variables that are not captured (eg, central vs peripheral tumor location). By concentrating on stage I tumors, we believe that we have minimized some of these effects (eg, clinical N1 disease) that can impact surgical approach, but we acknowledge that others persist.

To date there have been hundreds of VATS studies comparing thousands of lung cancer resections. The finding of fewer complications by the VATS approach appears to be consistent. As the volume of data increases, however, we must not ignore the persistence of potentially significant limitations. Consistency in the repetition of a flawed approach must not be mistaken for validation. Our study was specifically designed to improve and extend previous comparative strategies, rather than simply repeating them. We believe that our study strengthens the body of literature supporting VATS safety; however, there remain unanswered questions.

Recognizing individual variability in the VATS skill set, as well as potential variability relating to oncologic end points, it is difficult to declare a new criterion standard surgical approach to lung cancer. In the absence of a clinical trial, comparative effectiveness study of VATS versus thoracotomy will likely remain an imperfect science. We must be prepared for a point when available data resources have been maximally analyzed, scrutinized, and revised, and then we must either commit to the completion of a clinical trial or accept the trends in light of the residual limitations.

Daniel J. Boffa, MD

Section of Thoracic Surgery

Yale University School of Medicine

New Haven, Conn

http://dx.doi.org/10.1016/ j.jtcvs.2014.07.012

\section{CLINICAL CHALLENGES IN THE TREATMENT OF \\ PATIENTS WITH TRACHEOSTOMY IN A HYPERBARIC CHAMBER \\ To the Editor:}

Anastomotic separation is a rare but serious complication of tracheal repair. In a recent report, Stock and colleagues ${ }^{1}$ successfully used hyperbaric oxygen (HBO) therapy in patients with anastomotic complications after tracheal repair. We are pleased to see that the report by Stock and colleagues ${ }^{1}$ supports our previous findings, which showed that HBO therapy increases healing after tracheal resection and reconstruction in rats. $^{2}$ We hope that these 
observations, together, will encourage others to use HBO therapy in the management of this challenging clinical condition. Patients with tracheal repair who need tracheostomy, however, require special attention under HBO therapy. Some of the pertinent issues are discussed in the next paragraphs.

HBO therapy is the administration of $\sim 100 \%$ oxygen at higher atmospheric pressures in a pressurized vessel, the so-called hyperbaric chamber. There are 2 types of hyperbaric chambers: monoplace and multiplace. The former accommodates only 1 patient and is pressurized with oxygen; hence, the patient breathes oxygen directly from the surroundings. The latter accommodates multiple patients at the same time and is pressurized with air; therefore, patients breathe oxygen by way of a facemask, hood, or endotracheal tube.

Patients with tracheostomy experience increased respiratory distress inside the chamber owing to the increased density of the respired gases and the narrowing of their airway lumen by the tracheostomy. ${ }^{3}$ Because patients with tracheostomy cannot breathe through a facemask, 2 other options remain to deliver oxygen to patients treated in a multiplace chamber. The first option is to use a hood; however, most of the hoods will not be suitable for these patients because the neck seal of the hood corresponds to the tracheostomy opening. The second option is to attach a T-tube to the tracheostomy, which separately provides inspiration and expiration from the 2 limbs. However, this technique dramatically increases the respiratory resistance and makes breathing extremely hard for the patient. ${ }^{4}$ Therefore, monoplace chambers, which are compressed with oxygen and in which the patients breathe directly from the surroundings without the need for accessories, will be more suitable and comfortable for these patients.
Another important aspect of treating a patient with an artificial airway is the challenge of equalization of the middle ear pressure with the ambient pressure during the compression phase of HBO treatment. Failure to do so can lead to middle ear barotrauma, which is the most frequent side effect of HBO therapy. ${ }^{5}$ Patients with tracheostomy might require myringotomy or tympanostomy tube placement to avoid middle ear barotrauma, because they will not be able to efficiently auto-inflate their middle ear compartment. ${ }^{5}$

Finally, the tracheostomy tube cuff in patients undergoing $\mathrm{HBO}$ treatment should, preferably, be inflated with water rather than air. Air-filled cuffs will shrink at high pressure and cause the tracheostomy tube to be displaced.

We hope that the preventive measures we have noted will help to avoid HBO-related complications in patients with tracheostomy.

Gunalp Uzun, $M D$

Mesut Mutluoglu, MD

Department of Underwater and Hyperbaric Medicine

Gulhane Military Medical Academy Ankara, Turkey

\section{References}

1. Stock C, Gukasyan N, Muniappan A, Wright C, Mathisen D. Hyperbaric oxygen therapy for the treatment of anastomotic complications after tracheal resection and reconstruction. J Thorac Cardiovasc Surg. 2014;147:1030-5.

2. Gorur R, Hahoglu A, Uzun G, Kutlu A, Turut H, Yiyit N, et al. Effects of hyperbaric oxygen therapy on wound healing after tracheal resection and end-to-end anastomoses in rats: results of early observations. Thorac Cardiovasc Surg. 2008;56: 359-62.

3. Sieker HO, Saltzman HA. Medical considerations and applications of hyperbaric oxygenation. JAMA. 1965;193:31-6.

4. Prather ID, Wilson JR. An alternative oxygen supply technique for the difficult patient. Undersea Hyperb Med. 1995;22:183-4.

5. Presswood G, Zamboni WA, Stephenson LL, Santos PM. Effect of artificial airway on ear complications from hyperbaric oxygen. Laryngoscope. 1994;104:1383-4.

http://dx.doi.org/10.1016/ j.jtcvs.2014.07.012

\section{Reply to the Editor:}

Hyperbaric oxygen therapy (HBOT) is a promising therapy for the treatment of airway complications after tracheal surgery. In their letter to the Editor, Uzun and Mutluoglu describe its application specifically in patients who have a tracheostomy. Although we have not had any patients with tracheostomies requiring HBOT, we believe that anything to improve the safety and lessen the risk of complications of HBOT is a valuable contribution to the literature. Continued investigation into the benefits of HBOT for the treatment of airway complications after tracheal surgery is needed.

Cameron Stock, MD

Douglas Mathisen, MD

Massachusetts General Hospital Boston, Mass

http://dx.doi.org/10.1016/ j.jtcvs.2014.09.119

\section{FACTORS TO BE CONSIDERED IN AORTIC REPAIR \\ To the Editor:}

We read the article by Schäfers and colleagues ${ }^{1}$ with great interest. Because successful aortic valve repair aims at restoration of cusp and root dimensions, normal values of human cusps should be documented. The authors demonstrated that cusp height displays marked variability and is correlated with clinical variables. Furthermore, a positive correlation was observed between geometric cusp height and body height, the type of fusion (partial or complete) did not seem to have an effect on size of the cusp, and repair procedures may not provide a sufficient valvular stability for retracted valves. ${ }^{2}$ They also note that cusp height was larger than in previously published reports and suggest that their data could serve as a basis for decision making in aortic valve repair.

Prolapse and retraction of cusps and annular dilation are structural 